\section{Superconducting Mechanism of $\mathrm{MgB}_{2}$ Consistent with BCS Theory}

Researchers R. Osborn and colleagues at Argonne National Laboratory have confirmed that the superconducting mechanism of $\mathrm{MgB}_{2}$, a newly discovered noncopper-oxide superconductor with critical transition temperature $\left(T_{\mathrm{c}}\right)$ of $39 \mathrm{~K}$, can be explained by the Bardeen-CooperSchrieffer (BCS) theory. As reported in the June 18 issue of Physical Review Letters, the researchers performed inelastic neutronscattering measurement of the phonon density of states on an isotopically enriched polycrystalline sample of $\mathrm{Mg}^{11} \mathrm{~B}_{2}$, using time-of-flight neutron spectroscopy. They found that boron vibration frequencies extend up to energies of $100 \mathrm{meV}$. The acoustic phonons extend in energy to $36 \mathrm{meV}$, and there are highly dispersive optic branches peaking at $54,78,89$, and $97 \mathrm{meV}$. The frequencies of the main peaks are well represented by the Born-von Kármán force-constant model, and from which an electron-phonon coupling of $\sim 0.9$ was estimated from the Allen-Dynes equation. The measured values of the $T_{\mathrm{c}}$ and isotope effect are consistent with a conventional phonon mechanism for superconductivity with moderately strong electron-phonon coupling.

SHIMING WU

\section{$\mathrm{MgB}_{2}$ Crystallites Much Less Anisotropic than Cuprate HTS}

Relatively high values of critical current density $\left(J_{c}\right)$ and the absence of a grain-boundary weak-link problem have been observed for $\mathrm{MgB}_{2}$, making it a potentially useful material for superconductive devices. However, the layered lattice structure of $\mathrm{MgB}_{2}$ has made researchers wonder whether this compound is anisotropic like all the high $T_{\mathrm{c}}$ cuprates. In an article appearing in the June 25 issue of Physical Review Letters, direct measurements of upper critical field anisotropy and mass anisotropy ratio were made by O.F. de Lima and colleagues at UNICAMP in Brazil. The $\mathrm{MgB}_{2}$ sample was weakly sintered from a stoichiometric mixture of pure boron and pure magnesium powders. It was then crushed and milled to crystallites. Small amounts of the crystallites were $c$-axis aligned on paper. The real component of ac susceptibility was measured with an external magnetic field $H$ applied parallel and perpendicular to the sample $c$-axis.

The upper critical field $H_{\mathrm{C} 2}$, above which the superconductivity is destroyed, was calculated from the data taken in both orientations. The ratio between the upper critical field when $H$ is applied parallel to the $a b$ plane, and when it is along the $c$ direction, was evaluated at different temperatures, producing a ratio of about 1.7. This ratio, in turn, implies an anisotropy of the coherence length of 1.7 and a mass anisotropy ratio of 0.3 . Compared to the mass anisotropy ratio values found for the high $T_{\mathrm{c}}$ compounds Y-Ba-Cu-O (0.04) and Bi-Sr-Ca-Cu-O $\left(10^{-4}\right)$, both of which are known to be highly anisotropic materials, $\mathrm{MgB}_{2}$ is mildly anisotropic. In practical applications, the high $T_{\mathrm{c}}$ materials have to be textured to a maximum degree of $c$-axis alignment in order to obtain optimum $J_{c}$. While the texture might not be so important for the $\mathrm{MgB}_{2}$ devices, its reported anisotropy value indicates that the use of texturization techniques to optimize $J_{\mathrm{c}}$ in polycrystalline $\mathrm{MgB}_{2}$ would be advantageous.

SHIMING WU

\section{Composition "Libraries" Designed for Structural Materials}

High performance engineering applications require advanced structural materials, such as steels and superalloys, with properties superior to the constituent metals for the applications of interest. The high cost of traditional methods of iterative synthesis and testing greatly hinders progress in structural materials design. Ji-Cheng (J.-C.) Zhao has designed a technique to generate composition "libraries" for structural materials. As reported in a Communications in Advanced Engineering Materials 3 (2001) p. 143, Zhao first creates a "diffusion multiple" using thermal interdiffusion to form a bulk sample with continuous variations in multicomponent composition. Subsequently, he performs local measures of composition, phase, and properties across this sample, which provide an efficient chemical and mechanical survey of a range of multicomponent compositions. This technique is further described in a full-length article in Journal of Materials Research 16 (2001) p. 1565.

The concept of creating composition libraries is not new. Chemical libraries are perhaps best known for their applications in pharmaceutical drug design. More recently, combinatorial techniques have become increasingly popular for a wide variety of applications in functional materials.

Although alternative methods for determining solid-state continuous phase diagrams have been previously achieved, Zhao's method provides a significant step forward by allowing simultaneous determination of structural properties such as plastic hardness, toughness, and elastic stiffness - crucial features in materials applications. Previous methods using thin-film libraries cannot investigate such features because the thin-film nature of the sample often significantly alters the properties from those of the bulk materials.

This technique may offer significant potential for practical advances. If realized, not only would this technique allow faster concept-to-market turnaround in structural materials design, it should also permit more informed "fine-tuning" of complex materials composition. The nature of the diffusion sample allows investigation of a continuous multicomponent phase diagram rather than discrete modifications on sample tiles or production of macroscopic homogenous samples for analysis. This technique may also generate contributions in the realm of fundamental scientific interest. In particular, the rapid, in situ property measures may provide feedback very useful for modeling of complex materials.

Zhao initially applied his technique to study a Ni-NiAl diffusion couple. Armed with the success of this relatively simple test case, he went on to explore the much more complex, multicomponent sample $\mathrm{Ni}-\mathrm{Mo}-\mathrm{Fe}$, for which he plotted the multicomponent phase diagram and in situ measures of hardness and elastic modulus as a function of Mo content. Zhao ascertains that this method can be used to map multicomponent phase diagrams, look for novel compounds in multicomponent systems, determine diffusion $\mathrm{CO}^{-}$ efficients, evaluate precipitation kinetics, study the effects of compositional modifications in complex systems, and map composition-structure-property data.

"It is exciting to contemplate the advances this new technique will make possible," Zhao said. "This method should provide a significant speed-up over traditional synthesis and testing of structural materials."

EMILY JARVIS

\section{Separating and Probing Carbon Nanotubes for Advanced Nanoelectronics}

Carbon nanotubes, cylinders of graphene approximately 1-2 $\mathrm{nm}$ in diameter, may someday serve as interconnects in molecular electronics or as transistors that are 500 times smaller than current devices. Two teams of researchers-one from the IBM T.J. Watson Research Center in Yorktown Heights, NY, and the other from Harvard University in Cambridge, MAhave been separately investigating two of the fundamental issues associated with nanotubes to help achieve these goals. Both multiwalled nanotubes (MWNTs) and single-walled nanotubes (SWNTs) in bundles are complex conductors that incorporate many coupled tubes/shells that each 